\title{
Use of a xanthine oxidase free radical generating system to investigate the cytotoxic effects of reactive oxygen species on human spermatozoa
}

\author{
R. J. Aitken, D. Buckingham and D. Harkiss \\ MRC Reproductive Biology Unit, 37 Chalmers Street, Edinburgh EH3 9EW, UK
}

\begin{abstract}
The reaction between xanthine and xanthine oxidase results in the univalent and divalent reduction of dioxygen to generate superoxide $\left(\mathrm{O}_{2}^{-\bullet}\right)$ and hydrogen peroxide $\left(\mathrm{H}_{2} \mathrm{O}_{2}\right)$, respectively. With the aid of this system, the direct effect of reactive oxygen species (ROS) on human sperm function has been investigated. A protocol involving the addition of xanthine oxidase to the reaction mixture at 0 and $15 \mathrm{~min}$ resulted in a loss of motility involving every component of sperm movement examined. Lower doses of xanthine oxidase, which did not influence sperm motility, were also found to suppress the competence of human spermatozoa to exhibit oocyte fusion in response to the ionophore, A23187. The reactive oxygen species responsible for the disruption of human sperm function was not influenced by the presence of superoxide dismutase (SOD) or scavengers of hypochlorous acid or hydroxyl radicals. However, the cytotoxic species was shown to be extremely stable and could be completely eliminated by catalase, which selectively eliminates $\mathrm{H}_{2} \mathrm{O}_{2}$. Confirmation that it is $\mathrm{H}_{2} \mathrm{O}_{2}$, and not $\mathrm{O}_{2}^{-\bullet}$, which is cytotoxic to human spermatozoa was obtained in studies in which the direct addition of this oxidant was shown to influence both the movement of human spermatozoa and their competence for oocyte fusion. These results carry implications for the diagnosis of defective sperm function and the design of optimized culture media for the treatment of male factor infertility.
\end{abstract}

\section{Introduction}

Defective sperm function is a major cause of human infertility (Hull et al., 1985) and yet little is known of the aetiology of this condition or the precise nature of the defect(s) responsible for the loss of fertilizing potential. A significant, recent insight into this problem has emerged from an accumulation of evidence suggesting that peroxidative damage to the sperm plasma membrane is an important pathological mechanism in the onset of male infertility (Jones et al., 1979; Aitken and Clarkson, 1987a, b; Alvarez et al., 1987; Aitken et al., 1989a, b, 1991, 1992a; Iwasaki and Gagnon, 1992). Spermatozoa are particularly susceptible to such damage by virtue of their high content of unsaturated fatty acids (Jones et al., 1979) and their relative paucity of cytoplasmic enzymes for scavenging the reactive oxygen species that initiate lipid peroxidation (Alvarez et al., 1987; Alvarez and Storey, 1989; Jeulin et al., 1989).

The major sources of the oxidizing species that damage the spermatozoa are thought to be the spermatozoa themselves (Aitken and Clarkson, 1987a, b; Alvarez et al., 1987) and infiltrating leucocytes (Aitken and West, 1990; Kessopoulou et al., 1992), particularly neutrophils. However, despite the abundant evidence correlating the appearance of impaired sperm function with the generation of high amounts of reactive oxgyen species (ROS) by human spermatozoa (Aitken and Clarkson, 1987a, b; Aitken et al, 1989a, b, 1991, 1992a) or leucocytes (Wolff and Anderson, 1988a, b; Aitken and West, 1990; Kessopoulou et al, 1992), these data are only correlative.

Received 8 April 1992.
There have been no studies designed to evaluate the direct effect of ROS on human sperm function. Moreover, the identity of the ROS responsible for impairing sperm function has not been established. Although superoxide $\left(\mathrm{O}_{2}^{-\bullet}\right)$ appears to be the primary product of the free radical-generating system in human spermatozoa (Aitken and Clarkson, 1987a, b; Alvarez et al., 1987) a variety of secondary reactive oxygen species may arise through reactions involving dismutation, protonation and Fenton chemistry. A recent summary of the published literature concluded that $\mathrm{O}_{2}{ }^{-\bullet}$ rather than hydrogen peroxide $\left(\mathrm{H}_{2} \mathrm{O}_{2}\right)$ is the main inducer of lipid peroxidation in spermatozoa (Bize et al., 1991) and superoxide dismutase (SOD) has been proposed as a major determinant of the susceptibility of human spermatozoa to peroxidative damage (Alvarez et al., 1987). In the present study we have investigated these claims using an experimental system involving the exposure of purified human sperm suspensions to the powerful oxygen metabolites generated by xanthine oxidase in vitro. The results indicate that ROS can exert a direct, suppressive effect on many aspects of human sperm function and that $\mathrm{H}_{2} \mathrm{O}_{2}$ rather than $\mathrm{O}_{2}^{-} \cdot$ is the damaging species in such reactions.

\section{Materials and Methods}

\section{Spermatozoa}

The semen samples were obtained from normospermic donors (World Health Organization, 1987) and collected into sterile containers for immediate transportation to the laboratory. 
After allowing at least $30 \mathrm{~min}$ for liquefaction to occur, the spermatozoa were fractionated and purified on a discontinuous two-step Percoll gradient comprising $3 \mathrm{ml}$ of isotonic (100\%) Percoll overlaid with a further $3 \mathrm{ml}$ of $50 \%$ Percoll in a $10 \mathrm{ml}$ conical-based sterile centrifuge tube. Isotonic Percoll was created by supplementing $10 \mathrm{ml}$ of $10 \times$ concentrated Earles medium (Flow Laboratories, Irvine) with $300 \mathrm{mg}$ BSA, $3 \mathrm{mg}$ sodium pyruvate and $0.37 \mathrm{ml}$ of a sodium lactate syrup and adding $90 \mathrm{ml}$ of Percoll (Pharmacia, Uppsala). This preparation was designated $100 \%$ Percoll (Lessley and Garner, 1983) and was subsequently diluted by $50 \%$ with medium BWW (Biggers et al., 1971).

Semen $(1-2 \mathrm{ml})$ was layered on the top of each gradient and centrifuged at $500 \mathrm{~g}$ for $20 \mathrm{~min}$. Thereafter the seminal plasma was discarded and the cells collected from the base of the $100 \%$ Percoll fraction, resuspended in $7 \mathrm{ml}$ of medium BWW, centrifuged at $500 \mathrm{~g}$ for $5 \mathrm{~min}$ and finally resuspended at a sperm concentration of 20 or $100 \times 10^{6}$ spermatozoa ml $\mathrm{ml}^{-1}$, depending on the experiment being undertaken.

\section{Sperm function}

The movement characteristics of the spermatozoa were determined at $37^{\circ} \mathrm{C}$ in a Makler chamber (Sefi Medical Instruments, Haifa) using the Hamilton Thorn HT-M2030 Motility Analyser (Hamilton Thorn Research, Danvers, MA) in the $50 \mathrm{~Hz}$ PAL European format, incorporating version 7.0 software. System parameter settings for these analyses were 20 frames at 25 frames $\mathrm{s}^{-1}$; magnification factor 2.17 ; minimum contrast 12 ; minimum size 3; upper and lower gates of 0.4 and 1.6 for size and 0.5 and 2.0 for intensity; the default values for non-motile cells were 8 and 201 for size and intensity, respectively.

The analyses were consistently cross-checked using the Hamilton Thorn's playback facility to confirm the accuracy of the image analysis and, where necessary, adjustments were made to the parameter settings. The criteria of movement assessed in this study were curvilinear velocity (VCL - the twodimensional track described by the sperm head in unit time); progressive velocity (VSL - the straight line distance travelled by the sperm head in unit time); average path velocity (VAP the average path, calculated using a 5-point smoothing algorithm, travelled by the sperm head in unit time); percentage motile (the percentage of cells exhibiting a VAP of $>10 \mu \mathrm{m} \mathrm{s}^{-1}$ ); percentage rapid (the percentage of cells exhibiting a VAP of $>25 \mu \mathrm{m} \mathrm{s}^{-1}$ ) and ALH (the amplitude of lateral sperm head displacement in $\mu \mathrm{m})$.

The hamster oocyte penetration assay was conducted as previously described (Aitken and Clarkson, 1987a,b) except that the acrosome reaction was induced by exposure to 1.25 or $2.5 \mu \mathrm{mol} \mathrm{A23187} \mathrm{l}^{-1}$ for $2 \mathrm{~h}$. At the end of this period, the spermatozoa were pelleted by centrifugation at $500 \mathrm{~g}$ for $5 \mathrm{~min}$, resuspended in fresh BWW medium and distributed as $50 \mu \mathrm{l}$ droplets under liquid paraffin. Zona-free hamster oocytes were then introduced and incubated with the spermatozoa for a further $2 \mathrm{~h}$. The oocytes were finally washed free of adherent spermatozoa and compressed to a depth of about $30 \mu \mathrm{m}$ so that the incidence of decondensing sperm heads in the ooplasm could be determined by phase contrast microscopy. The results were expressed in terms of the percentage of oocytes penetrated and the mean number of penetrations per oocyte (total number of penetrations/total number of oocytes).
The acrosome reaction was assessed using the method described by Mortimer et al. (1988) using fluorescein-conjugated peanut lectin to label the outer acrosomal membrane. The viability of the spermatozoa used in the acrosome reaction test was assessed by prior exposure of the spermatozoa to a hypo-osmotic swelling medium for 1 h (Jeyendran et al., 1984). As a consequence of this treatment, viable spermatozoa exhibited a marked curling of the tail and only these cells were scored in assessing the acrosome reaction rate.

\section{Reactive oxygen species}

Reactive oxygen species were generated using the xanthine: xanthine oxidase system described by McCord and Fridovich (1968). Xanthine oxidase catalyses the univalent and divalent reduction of ground state oxygen to generate both $\mathrm{O}_{2}^{-} \bullet$ and $\mathrm{H}_{2} \mathrm{O}_{2}$ and results in the oxidation of xanthine to uric acid. In addition to the generation of $\mathrm{O}_{2}{ }^{-\bullet}$ and $\mathrm{H}_{2} \mathrm{O}_{2}$, secondary radicals, such as the highly reactive hydroxyl radical $\left(\mathrm{OH}^{\bullet}\right)$, may form through the catalytic action of transition metals, particularly iron, in a Haber-Weiss reaction. The presence of trace amounts of iron in the medium also has a direct inactivating effect on xanthine oxidase through the creation or exposure of reactive sulfur centres upon reduction of the enzyme by its substrates. These complications due to the presence of transition metals were avoided by incorporating into the free radical-generating system used in this study the chelating agent diethylene triamine pentacetic acid (DETAPAC), which has been shown to be more effective than desferrioxamine in limiting the production of $\mathrm{OH}^{\bullet}$ by the metal-catalysed Haber-Weiss reaction in the xanthine oxidase system (Britigan et al., 1990).

Stock solutions of $1.32 \mathrm{mmol}$ DETAPAC $\mathrm{I}^{-1}(0.026 \mathrm{~g}$ in $50 \mathrm{ml}$ medium BWW $)$ and $17.8 \mathrm{mmol}$ xanthine $\mathrm{I}^{-1}(0.062 \mathrm{~g}$ in $20 \mathrm{ml}$ Hanks balanced salt solution) were prepared and mixed to give final concentrations of $1.1 \mathrm{mmol}$ DETAPAC $\mathrm{l}^{-1}$ and $2 \mathrm{mmol}$ xanthine $1^{-1}$. The generation of reactive oxygen species was initiated by the addition of xanthine oxidase, at the concentrations stipulated in the results section. Superoxide dismutase (SOD; Calbiochem, San Diego, CA, USA) and catalase (Calbiochem) were used to remove $\mathrm{O}_{2}^{-\bullet}$ or $\mathrm{H}_{2} \mathrm{O}_{2}$ from the system, respectively. The SOD exhibited a specific activity of $3000 \mathrm{U} \mathrm{mg}^{-1}$ dry weight and was prepared daily as a $1 \mathrm{mg} \mathrm{ml} \mathrm{m}^{-1}$ stock solution in medium BWW. The catalase (5000 $\mathrm{U} \mathrm{mg}^{-1}$ dry weight) was also prepared daily in medium BWW at a concentration of $1 \mathrm{mg}$ in $1.5 \mathrm{ml}$. Unless otherwise indicated the concentration of SOD and catalase in the reaction mixture to which the spermatozoa were added was $100 \mathrm{U} \mathrm{ml}^{-1}$.

Chemiluminescence. The activity of the free radicalgenerating system was assessed by monitoring the generation of $\mathrm{O}_{2}^{-} \cdot$ by lucigenin-dependent chemiluminescence (Gyllenhammer 1987). Lucigenin was made up as a $25 \mathrm{mmol} \mathrm{l}^{-1}$ stock solution in dimethyl sulfoxide and added to the reaction mixtures used in this study at a final concentration of $250 \mu \mathrm{mol} \mathrm{I}^{-1}$. The chemiluminescent response was measured on a Berthold Biolumat LB9500T luminometer in the integration mode.

Spectrophotometry. Measurement of the $\mathrm{O}_{2}^{-} \cdot$ generated during the xanthine oxidase reaction was achieved using nitro 
blue tetrazolium (NBT) as described by Green and Hill (1984) $100 \mathrm{U}$ SOD ml ${ }^{-1}$ being added to the control incubations. The amount of hydrogen peroxide generated in the xanthine oxidase system was measured using the peroxidase catalysed oxidation of 4-aminoantipyrine in the presence of phenol (Green and Hill, 1984). For this assay, a reagent mix was created containing $25 \mathrm{mmol}$ phenol $\mathrm{l}^{-1}$ and $5 \mathrm{mmol} \mathrm{4-aminoantipyrine}$ $1^{-1}$ in $100 \mathrm{ml}$ distilled water to which $\mathrm{Iml} 100 \mathrm{mmol} \mathrm{K}_{3} \mathrm{PO}_{4} \mathrm{I}^{-1}$ containing $8 \mathrm{mg}$ horseradish peroxidase $\mathrm{ml}^{-1}$ and $2.5 \mu \mathrm{mol}$ $\mathrm{H}_{2} \mathrm{O}_{2} \mathrm{I}^{-1}$ were added. The specificity of the assay was assured by incorporating $100 \mathrm{U}$ catalase $\mathrm{ml}^{-1}$ into the control incubations. Sample or $\mathrm{H}_{2} \mathrm{O}_{2}$ standard, $20 \mu \mathrm{l}$, was pipetted into the well of a microtitre plate and mixed with $100 \mu$ l of distilled water and $80 \mu \mathrm{l}$ of the reagent mix. The change in absorbance at $510 \mathrm{~nm}$ was then monitored and the $\mathrm{H}_{2} \mathrm{O}_{2}$ concentrations determined.

Fluorimetry. $\mathrm{H}_{2} \mathrm{O}_{2}$ was also assayed in a fluorometric assay using homovanillic acid as substrate. The reaction mixture contained $500 \mu \mathrm{l}$ spermatozoa at $10 \times 10^{6} \mathrm{ml}^{-1}, 15 \mu \mathrm{l}$ of a $100 \mu \mathrm{mol} 1^{-1}$ solution of homovanillic acid and $6 \mu \mathrm{l}(3.7$ purpurogallin units) of horseradish peroxidase; the excitation and emission wavelengths used were 315 and $425 \mathrm{~nm}$, respectively.

\section{Lipid peroxidation}

The influence of the reactive oxygen species generated by the xanthine oxidase system on the peroxidation status of the spermatozoa was monitored using the generation of malondialdehyde as the end-point. Malondialdehyde was assessed using the thiobarbituric acid method (Slater, 1984). For this assay, spermatozoa that had previously been isolated by Percoll gradient centrifugation were pelleted by centrifugation and resuspended at a concentration of $10 \times 10^{6} \mathrm{ml}^{-1}$ in Hanks balanced salt solution containing no magnesium or calcium. Where indicated, the breakdown of lipid peroxides to yield malondialdehyde was accelerated by the addition of a ferrous ion catalyst $\left(40 \mu \mathrm{mol}\right.$ ferrous sulphate $\mathrm{I}^{-1}$ and $200 \mu \mathrm{mol}$ ascorbate $\left.1^{-1}\right)$. A reaction mixture was created comprising $200 \mu \mathrm{l} 7 \% \mathrm{SDS}, 2 \mathrm{ml} 0.1 \mathrm{~mol} \mathrm{HCl} \mathrm{l}^{-1}, 300 \mu \mathrm{l} 10 \%$ phosphotungstic acid, $100 \mu \mathrm{l} 0.2 \mathrm{mmol}$ butylated hydroxytoluene $\mathrm{l}^{-1}$ and $1 \mathrm{ml} 0.67 \%$ 2-thiobarbituric acid. This assay mixture, $360 \mu \mathrm{l}$, was added to $200 \mu \mathrm{l}$ of spermatozoa and boiled for $30 \mathrm{~min}$ in a water bath. Once the tubes had cooled, $500 \mu \mathrm{l}$ of butanol was mixed with the solution and the upper layer removed for analysis in a spectrofluorimeter using excitation and emission wavelengths of 510 and $553 \mathrm{~nm}$, respectively. Standards were generated by the acid hydrolysis of $1,1,3,3$ tetraethoxypropane in $0.1 \mathrm{~mol} \mathrm{HCl}^{-1}$.

\section{Statistical analysis}

All experiments were repeated at least three times, the exact number of replicates being indicated in the text. The statistical significance of the results was assessed using analysis of variance (ANOVA) with repeated measures using the Statview program (Abacus Concepts, Berkeley, CA) on an Apple Macintosh SE30 computer. This procedure generated an overall significance value for the differences due to a particular variable such as time or treatment but, in addition, offered the potential to examine the differences between individual group means using a threshold significance value set at $P<0.05$.

\section{Results}

\section{Characterization of the xanthine oxidase system}

A series of preliminary experiments were conducted in which different regimens of xanthine oxidase administration were compared for their capacity to induce a prolonged period of oxidative stress. As a consequence of these studies, a $30 \mathrm{~min}$ regimen involving two administrations of xanthine oxidase $(0.04 \mathrm{U})$ at $t=0$ and $t=15 \mathrm{~min}$ were identified as optimal. The biphasic profile of $\mathrm{O}_{2}{ }^{-}$and $\mathrm{H}_{2} \mathrm{O}_{2}$ generated under these conditions was monitored by NBT reduction and homovanillic acid oxidation, respectively (Fig. 1). Measurements of the concentration of each of these ROS in the medium at the end of the $30 \mathrm{~min}$ incubation revealed the presence of $219.9 \pm$ $1.9 \mu$ mol catalase-inhibitable $\mathrm{H}_{2} \mathrm{O}_{2} \mathrm{l}^{-1}$ and $25.5 \pm 2.3 \mu \mathrm{molSOD}$ inhibitable $\mathrm{O}_{2}^{-\bullet} \mathrm{I}^{-1}$, respectively. The concentration of $\mathrm{O}_{2}^{-} \bullet$ was found to be significantly increased to $27.6 \pm 2.2 \mu \mathrm{mol} 1^{-1}$, by the presence of $100 \mathrm{U}$ catalase $\mathrm{ml}^{-1}(P<0.5 ; n=12)$, whereas the $\mathrm{H}_{2} \mathrm{O}_{2}$ signal was significantly increased by the presence of $100 \mathrm{U}$ SOD ml ${ }^{-1}$ to $233.9 \pm 2.5 \mu \mathrm{mol} \mathrm{^{-1 }}$ $(P<0.05 ; n=12)$.

The kinetics of reactive oxygen species generation in the xanthine oxidase system could be determined more accurately by chemiluminescence, since this is a non-integrative technique which responds very rapidly to changes in the rate at which a given metabolite is generated. Peroxidase-enhanced, luminoldependent chemiluminescence was initially used in an attempt to monitor the kinetics of $\mathrm{H}_{2} \mathrm{O}_{2}$ generation in the xanthine oxidase system (Wymann et al, 1987; Vilim and Wilhelm, 1989; Aitken et al., 1992b). However, the results of this analysis (Fig. 1) revealed that after the induction of an instantaneous burst of chemiluminescence in response to the initial administration of xanthine oxidase, a second administration of oxidase at $15 \mathrm{~min}$ invoked a barely detectable response, due to the scavenging effects of uric acid produced as a consequence of xanthine oxidation (Radi et al., 1990). Moreover, the system lacked specificity for $\mathrm{H}_{2} \mathrm{O}_{2}$ since $\mathrm{SOD}$, but not catalase, could suppress the luminol-dependent chemiluminescent signal. Such interference from the product of xanthine oxidation, coupled with the lack of specificity, meant that this particular chemiluminescence system could not be used to supplement the fluorimetry data in describing the kinetics of $\mathrm{H}_{2} \mathrm{O}_{2}$ generation.

In contrast, lucigenin-dependent chemiluminescence was effective in monitoring $\mathrm{O}_{2}{ }^{-} \cdot$ generation during the $30 \mathrm{~min}$ incubation (Gyllenhammar, 1987; Fig. 1). Immediately after the primary addition of xanthine oxidase, the lucigenin-signal increased to reach a plateau after $10 \mathrm{~min}$ followed by a second short-lived peak in response to the subsequent addition of oxidase at $15 \mathrm{~min}$ (Fig. 1). The addition of SOD, alone or in the presence of catalase, completely suppressed this lucigenindependent signal, confirming that this probe was responding to the generation of $\mathrm{O}_{2}{ }^{-} \cdot$. In contrast, addition of catalase resulted in an increase in the $\mathrm{O}_{2}{ }^{-\bullet}$ signal, as noted above. This response was characterized by a sudden rise in chemiluminescence $5 \mathrm{~min}$ 

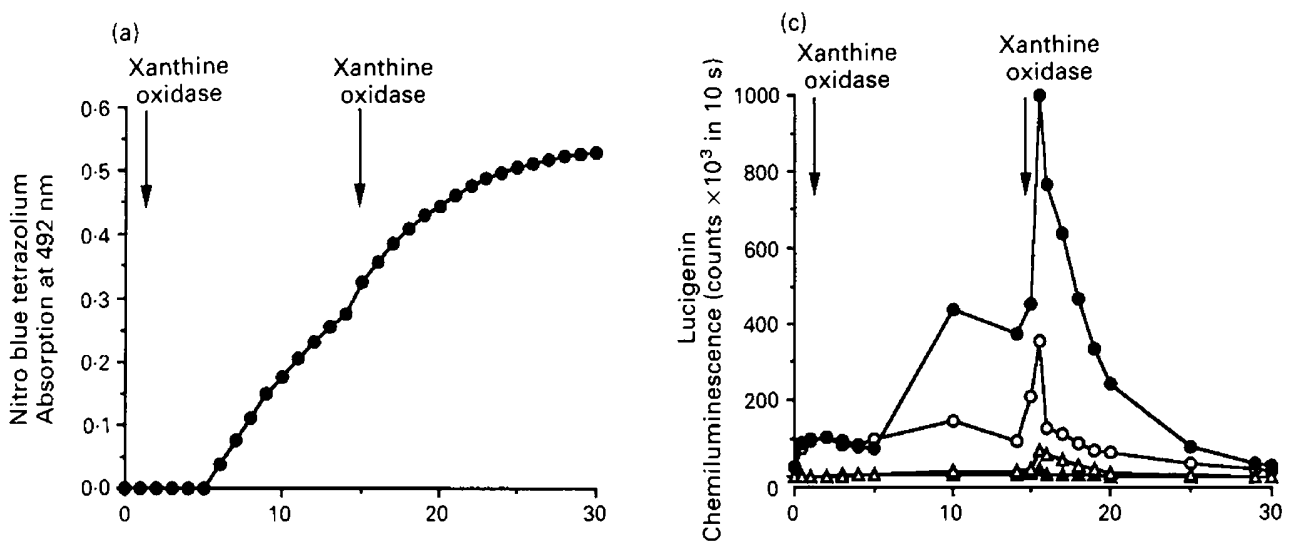

(b)
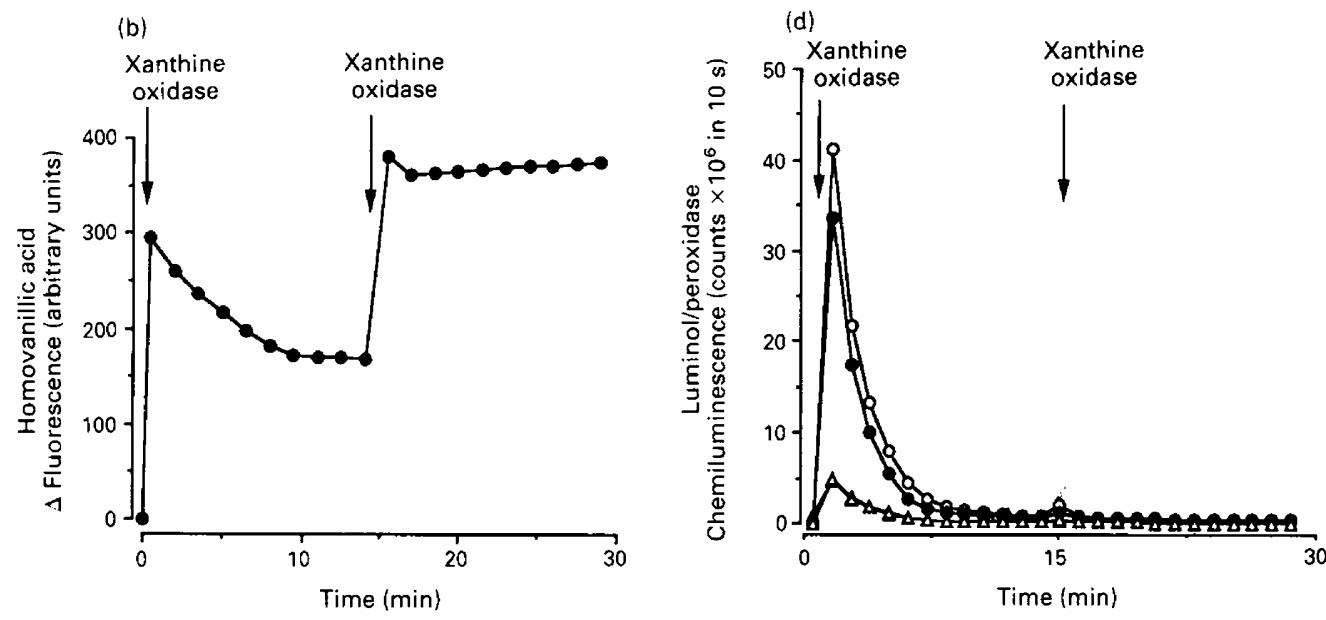

Fig. 1. Kinetics of reactive oxygen species generation in the xanthine oxidase system used in this study by (a) nitro blue tetrazolium reduction for monitoring $\mathrm{O}_{2}{ }^{-}$, (b) homovanillic acid oxidation for monitoring $\mathrm{H}_{2} \mathrm{O}_{2}$, ordinate axis describes the change in fluorescence from the baseline at zero time (c) lucigenin-dependent chemiluminescence for accurate time resolution of $\mathrm{O}_{2}{ }^{-}$response kinetics and (d) luminol- and peroxidase-dependent chemiluminescence for time resolution of $\mathrm{H}_{2} \mathrm{O}_{2}$ generation but revealing marked inhibition of the chemiluminescent signal as a consequence of uric acid generation. For $(\mathrm{c})$ and $(\mathrm{d})(\mathrm{O})$ - catalase, $-\mathrm{SOD} ;(\mathbf{O})+$ catalase, $-\mathrm{SOD} ;(\boldsymbol{\Delta})$ - catalase, +SOD; $(\triangle)+$ catalase, +SOD. Plots are representative of three independent experiments, all of which showed the same pattern.

after the primary addition of oxidase and a second major peak immediately after the subsequent administration of this enzyme at $15 \mathrm{~min}$ (Fig. 1).

\section{Sperm movement characteristics}

The influence of these different profiles of reactive oxygen species generation on the movement characteristics of human spermatozoa was assessed by taking measurements at 0,15 and $30 \mathrm{~min}$. In the absence of any scavenging enzymes, the spermatozoa responded to the oxidative stress created by the xanthine oxidase system with a progressive and significant $(P<0.01$ for the overall effect of time) decline in percentage motility (Fig. 2) and every criterion of sperm movement assessed (VAP, VCL, VSL, ALH and \% rapid) over the 30-min experiment. In Table 1 , the data for VCL $(P=0.01) \mathrm{ALH}(P=0.01)$ and $\%$ rapid $(P=$ 0.0003 ) are given as examples. In the presence of SOD, an even more significant decline of sperm movement was observed
$(P=0.0002$ for VCL; $P=0.001$ for ALH and $P=0.0001$ for $\%$ rapid), despite the fact that $\mathrm{O}_{2}{ }^{-\bullet}$ generation was suppressed under these conditions (Table 1). However, in the presence of catalase, alone or in combination with SOD, the movement characteristics were unaltered by exposure to the xanthine oxidase system (Table 1). In the case of the amplitude of lateral sperm head displacement, the presence of catalase was even found to have significantly increased this attribute of sperm movement over the $30 \mathrm{~min}$ incubation $(P<0.05$; Table 1$)$. Comparison of the movement characteristics exhibited by the spermatozoa in the presence of SOD plus catalase or catalase alone did not reveal any significant differences, with the exception of percentage motility, which was slightly higher in the presence of catalase alone $(91.2 \pm 1.9 \%$ versus $82.25 \pm 1.7 \%$ ).

Catalase was the only scavenging reagent assessed that exhibited a capacity to rescue spermatozoa from the cytotoxic effects of reactive oxygen species generated by the xanthine 

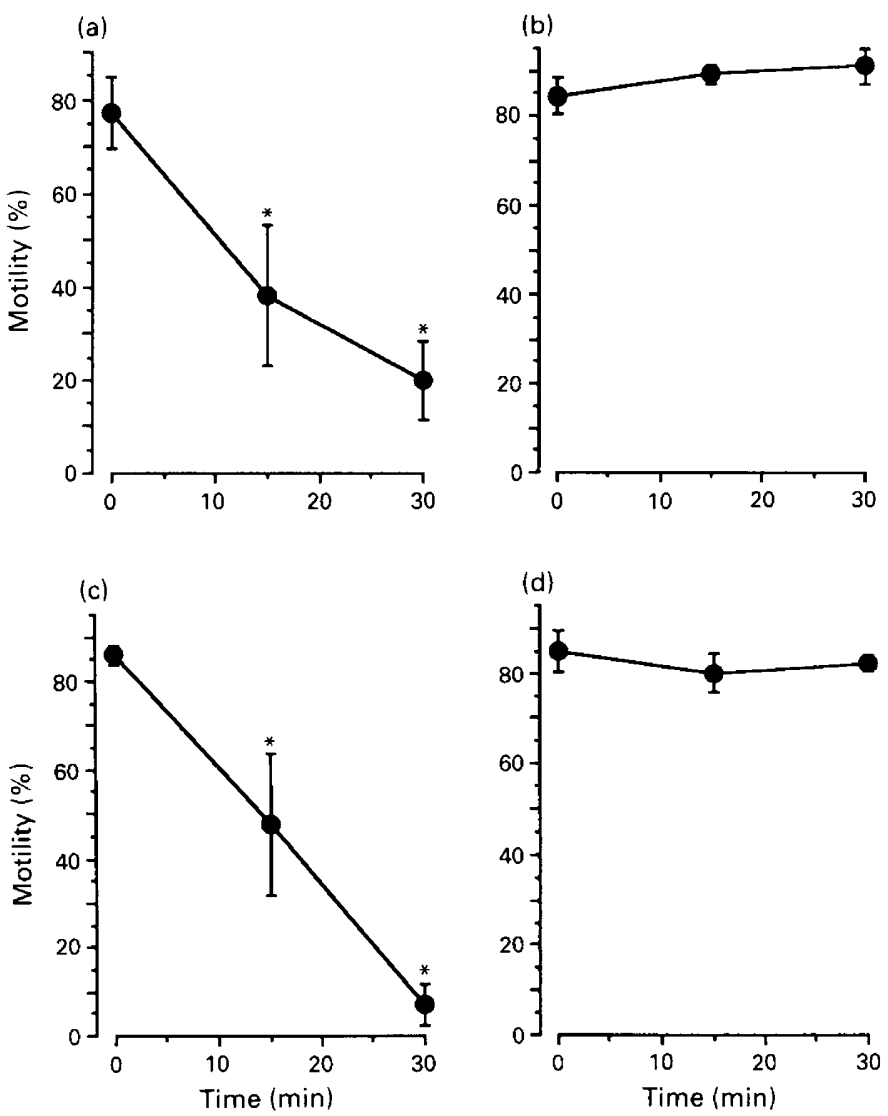

Fig. 2. Changes in the percentage of motile spermatozoa in response to the reactive oxygen species generated by the xanthine oxidase system. Note the ability of catalase to rescue this component of sperm movement, $n=4{ }^{*} P<0.05$ compared with motility at time zero.

Table 1. Influence of the xanthine oxidase system on the movement characteristics of human spermatozoa

Movement characteristic

Curvilinear velocity $\left(\mu \mathrm{m} \mathrm{s}^{-1}\right)$

Rapid (\%)

Amplitude of lateral head displacement $(\mu \mathrm{m})$
Treatment

Control

SOD

CAT

$\mathrm{SOD}+\mathrm{CAT}$

Control

SOD

CAT

$\mathrm{SOD}+\mathrm{CAT}$

Control

SOD

CAT

$\mathrm{SOD}+\mathrm{CAT}$
Time (min)

\begin{tabular}{ccc}
\hline 0 & 15 & 30 \\
$68.5 \pm 5.9$ & $38.0 \pm 6.2^{*}$ & $20.2 \pm 7.3^{*}$ \\
$87.7 \pm 6.5$ & $29.0 \pm 2.5^{*}$ & $13.7 \pm 5.9^{*}$ \\
$78.5 \pm 5.0$ & $85.5 \pm 5.7$ & $86.2 \pm 2.9$ \\
$79.2 \pm 11.5$ & $88.0 \pm 4.5$ & $79.5 \pm 4.7$ \\
$59.2 \pm 10.0$ & $9.5 \pm 3.7^{*}$ & $6.0 \pm 3.7^{*}$ \\
$77.0 \pm 2.3$ & $10.7 \pm 3.3^{*}$ & $3.2 \pm 3.3^{*}$ \\
$65.7 \pm 4.3$ & $79.7 \pm 3.3$ & $85.2 \pm 2.6$ \\
$74.2 \pm 8.3$ & $76.0 \pm 4.4$ & $73.2 \pm 1.5$ \\
$4.1 \pm 0.5$ & $2.2 \pm 0.3^{*}$ & $1.2 \pm 0.5^{*}$ \\
$4.5 \pm 0.6$ & $1.5 \pm 0.5^{*}$ & $0.3 \pm 0.2^{*}$ \\
$3.5 \pm 0.2$ & $4.5 \pm 0.5$ & $4.9 \pm 0.1$ \\
$4.2 \pm 0.6$ & $4.5 \pm 0.4$ & $4.5 \pm 0.4$
\end{tabular}

'Percentage of cells exhibiting an average path velocity $>25 \mu \mathrm{m} \mathrm{s}^{-1}$.

${ }^{*} P=<0.05$ compared with zero time.

Number of replicates $=4$.

SOD: superoxide dismutase; CAT: catalase. 
Table 2. Inhibition of sperm movement following the addition of spermatozoa to the xanthine oxidase system, $1 \mathrm{~h}$ after the initiation of reactive oxygen species generation with xanthine oxidase

\begin{tabular}{|c|c|c|c|c|c|}
\hline \multirow[b]{2}{*}{$\begin{array}{l}\text { Movement } \\
\text { characteristic }\end{array}$} & \multicolumn{5}{|c|}{ Treatment } \\
\hline & Control & XO & $X O+S O D$ & $\mathrm{XO}+\mathrm{CAT}$ & $\begin{array}{c}\mathrm{XO}+\mathrm{SOD} \\
+\mathrm{CAT}\end{array}$ \\
\hline Motility (\%) & $72.7 \pm 4.3$ & $1.0 \pm 1.0^{*}$ & $0.0 \pm 0.0^{*}$ & $71.3 \pm 4.2$ & $69.3 \pm 6.7$ \\
\hline $\begin{array}{l}\text { Curvilinear velocity } \\
\left(\mu \mathrm{m} \mathrm{s}^{-1}\right)\end{array}$ & $86.3 \pm 6.0$ & $3.7 \pm 3.7^{*}$ & $0.0 \pm 0.0^{*}$ & $89.0 \pm 3.8$ & $93.3 \pm 2.3$ \\
\hline
\end{tabular}

${ }^{*} P=<0.05$ compared with control.

Number of replicates $=3$.

$\mathrm{XO}$ : xanthine oxidase; SOD: superoxide dismutase; CAT: catalase.

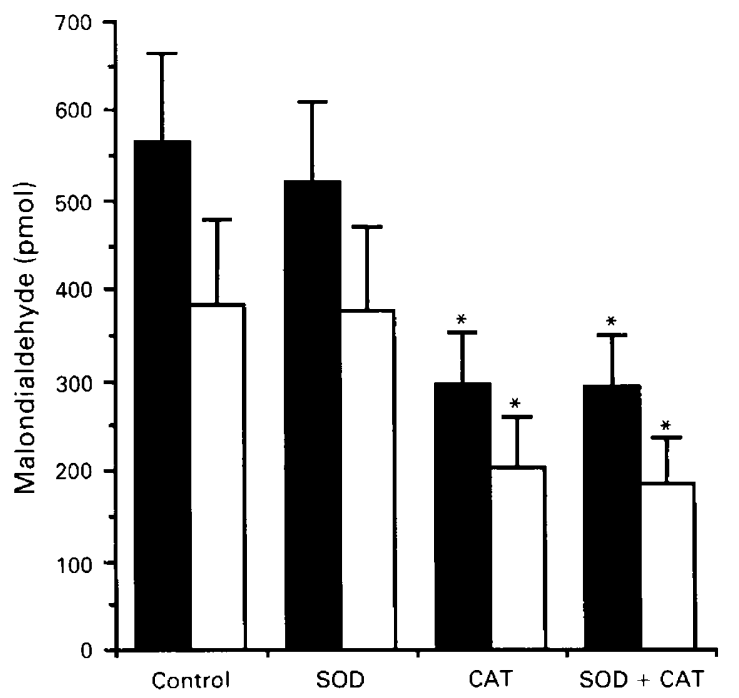

Fig. 3. Lipid peroxidation in spermatozoa induced by reactive oxygen species generated by the xanthine oxidase system. CAT: catalase; SOD: superoxide dismutase, $n=6$. ( $\square$ ) + ferrous ion promoter, ( $\square$ ) -ferrous ion promoter. ${ }^{*} P<0.05$ compared with control.

oxidase system. Inclusion of additional chelation reagents ( $2 \mathrm{mmol}$ desferrioxamine $\mathrm{l}^{-1}$ ), inactivators of hypochlorous acid $\left(2 \mathrm{mmol}\right.$ taurine $\left.\mathrm{I}^{-1}\right)$, or scavengers of hydroxyl radicals $\left(20 \mathrm{mmol}\right.$ mannitol $\left.\mathrm{l}^{-1}\right)$, did not reverse the decline in sperm motility observed in the presence of xanthine oxidase (data not shown).

The reactive oxygen species responsible for the loss of sperm movement were long lived. This was ascertained in a modified experimental design in which the spermatozoa were absent from the reaction mixture during the additions of xanthine oxidase and were added only one hour after the initiation of oxidant generation. The spermatozoa were then assessed after $30 \mathrm{~min}$ exposure to the long-lived products of the oxidase system, to determine whether their motility had been affected. The results of this experiment, illustrated by reference to the VCL and motility data given in Table 2, indicate that the oxidative species responsible for attacking the spermatozoa persisted in the reaction mixture for at least $60 \mathrm{~min}$ after the primary addition of oxidase. This result ruled out the possibility that short-lived
Table 3. Influence of the xanthine oxidase system on the capacity of human spermatozoa for sperm-oocyte fusion

\begin{tabular}{lcc}
\hline & $\begin{array}{c}\text { Oocyte } \\
\text { penetration } \\
(\%)\end{array}$ & $\begin{array}{c}\text { Polyspermy } \\
\text { (sperm per oocyte) }\end{array}$ \\
\hline Treatment & $98.8 \pm 1.2$ & $7.1 \pm 0.9$ \\
Control & $73.8 \pm 10.8^{*}$ & $2.2 \pm 1.0^{*}$ \\
$\mathrm{XO}+\mathrm{CAT}$ & $100.0 \pm 0.0$ & $6.7 \pm 1.5$ \\
$\mathrm{XO}+\mathrm{SOD}$ & $69.4 \pm 10.1^{*}$ & $1.6 \pm 0.4^{*}$ \\
$\mathrm{XO}+\mathrm{CAT}+\mathrm{SOD}$ & $94.3 \pm 3.9$ & $4.2 \pm 1.1$ \\
\hline
\end{tabular}

${ }^{*} P=<0.05$ compared with respect to control.

Number of replicates $=6$.

XO: xanthine oxidase; SOD: superoxide dismutase; CAT: catalase.

radicals (for example $\mathrm{OH}^{\bullet}$ ) could have been responsible for the loss of motility. The concept that a long-lived oxidant such as $\mathrm{H}_{2} \mathrm{O}_{2}$ could have remained in solution and inhibited sperm motility was supported by the beneficial effect of catalase, but not SOD, in this experiment (Table 2). Confirmation that $\mathrm{H}_{2} \mathrm{O}_{2}$ is a cytotoxic species with respect to human spermatozoa was obtained in dose-dependent studies in which the direct addition of this oxidant to suspensions of human spermatozoa was found to suppress sperm movement at concentrations above $500 \mu \mathrm{mol}]^{-1}$ (data not shown).

\section{Lipid peroxidation}

The beneficial effect of catalase, relative to SOD, was also revealed by an analysis of lipid peroxidation in response to the reactive oxygen species generated by the xanthine oxidasexanthine combination. Exposure of human spermatozoa to the xanthine oxidase system resulted in the induction of significant lipid peroxidation, reflected in the generation of high amounts of malondialdehyde. The addition of a ferrous iron promotor to the system increased the amount of malondialdehyde detected by catalysing the breakdown of lipid peroxides in the spermatozoa (Fig. 3). The presence of catalase, but not SOD, in the reaction mixture significantly suppressed $(P<0.01)$ the degree 

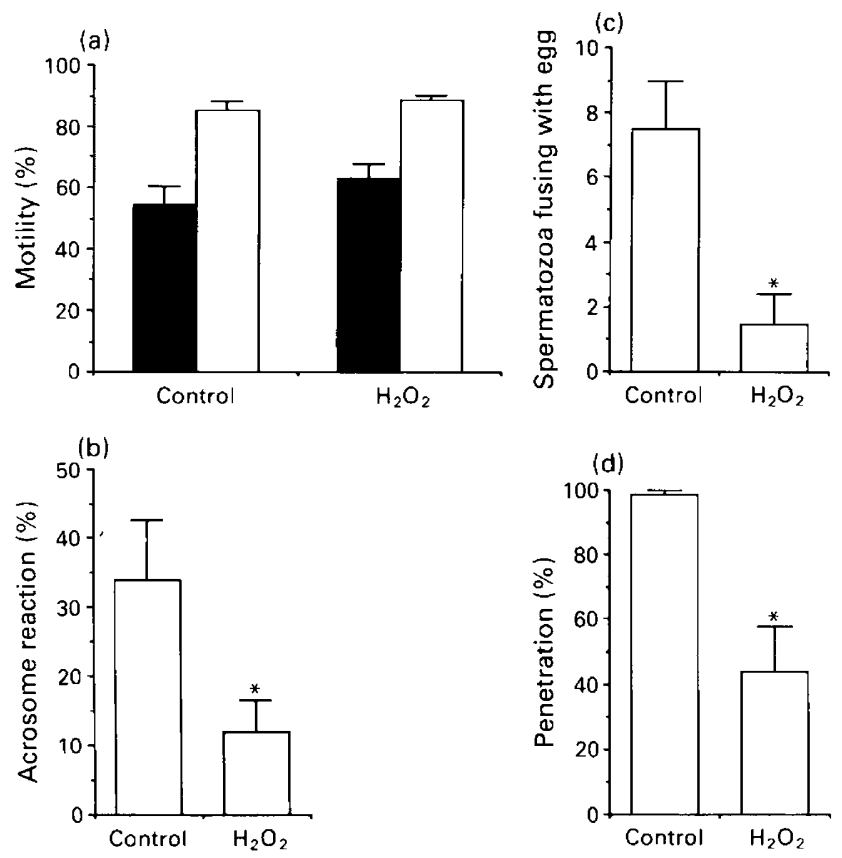

Fig. 4. Direct effect of $200 \mu \mathrm{mol} \mathrm{H}_{2} \mathrm{O}_{2} l^{-1}$ on the rate of sperm-oocyte fusion in the zona-free hamster oocyte assay. (a) $\mathrm{H}_{2} \mathrm{O}_{2}$ did not affect the motility ( $\boldsymbol{\square}$ ) or viability ( $\square$ ) of the spermatozoa; (b) $\mathrm{H}_{2} \mathrm{O}_{2}$-induced inhibition of the acrosome reaction; (c) $\mathrm{H}_{2} \mathrm{O}_{2}$-induced inhibition of sperm-oocyte fusion expressed as mean number of spermatozoa: egg; (d) $\mathrm{H}_{2} \mathrm{O}_{2}$-induced inhibition of sperm-oocyte fusion expressed as percentage penetration, $n=6 .{ }^{*} P<0.05$ compared with control.

of lipid peroxidation induced by the xanthine oxidase system, as measured in the presence or absence of promotor (Fig. 3).

\section{Hamster oocyte fusion and acrosome reaction}

The influence of reactive oxygen species on the capacity of human spermatozoa for sperm-oocyte fusion was assessed by exposing these cells to the xanthine oxidase system for $30 \mathrm{~min}$ before centrifugation $(5 \mathrm{~min}$ at $500 \mathrm{~g}$ ) and resuspension in fresh BWW medium containing $1.25 \mu \mathrm{mol} \mathrm{A} 23187 \mathrm{l}^{-1}$ as the free acid. After a further $2 \mathrm{~h}$ incubation in the presence of ionophore, the spermatozoa were once again pelleted by centrifugation $(5 \mathrm{~min}$ at $500 \mathrm{~g})$, resuspended in BWW and dispersed as $50 \mu \mathrm{l}$ droplets under liquid paraffin, in preparation for the zona-free hamster oocyte penetration test. Since the capacity of human spermatozoa to fuse with the vitelline membrane of the oocyte is highly dependent on the concentration of motile spermatozoa (Aitken and Elton, 1984, 1986), the concentration of xanthine oxidase was reduced to a level at which the percentage of motile spermatozoa was unaltered. Dose-response studies indicated that the optimal regimen involved a $30 \mathrm{~min}$ exposure to the ROS generated by the addition of $50 \mu$ l aliquots of xanthine oxidase $\left(0.0287 \mathrm{U} \mathrm{ml}^{-1}\right)$ at 0 and $15 \mathrm{~min}$. The concentration of $\mathrm{H}_{2} \mathrm{O}_{2}\left(53.99 \pm 0.35 \mu \mathrm{mol} \mathrm{l}^{-1}, n=12\right)$ detected in the medium at the end of the $30 \mathrm{~min}$ exposure period was not associated with any change in the percentage of motile spermatozoa between the various treatment groups, after the spermatozoa had been washed, incubated with A23187 and prepared for hamster oocyte fusion (control $40.8 \pm 7.2 \%$; catalase $38.0 \pm 8.1 \%$; SOD $40.7 \pm 7.4 \%$ and SOD plus catalase $41.3 \pm 8.4 \%$ ). Despite this equivalence of sperm motility, considerable differences in the levels of sperm-oocyte fusion were observed between groups (Table 3). Whether the results were expressed in terms of the percentage of oocytes penetrated or the degree of polyspermy (mean number of spermatozoa penetrating each oocyte), exposure to the xanthine oxidase system was found to induce a significant decline $(P<0.05)$ in the level of sperm-oocyte fusion. A similar decline was observed in the incubations supplemented with SOD. However the xanthine oxidase system had no significant effect on the levels of sperm-oocyte fusion observed when catalase was present in the reaction mixture prior to the addition of A23187, whether or not SOD was also present (Table 3).

The beneficial effects of catalase on the rates of spermoocyte fusion observed in the presence of the xanthine oxidase system suggested that $\mathrm{H}_{2} \mathrm{O}_{2}$ was instrumental in suppressing this aspect of sperm function. To confirm that this was the case, the direct effect of $\mathrm{H}_{2} \mathrm{O}_{2}$ on sperm-oocyte fusion was examined by administering this oxidant at a concentration that did not influence sperm motility or viability. Dose-dependent studies established that the incubation of human spermatozoa for $3 \mathrm{~h}$ following the administration of $200 \mu \mathrm{mol} \mathrm{H}_{2} \mathrm{O}_{2} \mathrm{1}^{-1}$, with $2.5 \mu \mathrm{mol} \mathrm{A} 23187 \mathrm{l}^{-1}$ present for the last $2.5 \mathrm{~h}$, was fully compatible with the maintenance of motility and viability (Fig. 4). In this situation, the control samples exhibited high rates of sperm-oocyte fusion and acrosome reaction in response to the second messengers induced by the ionophore, whereas the spermatozoa treated with $\mathrm{H}_{2} \mathrm{O}_{2}$ exhibited significantly diminished responses in both bioassays of sperm function (Fig. 4).

\section{Discussion}

The xanthine oxidase system used in this study generated a mixture of $\mathrm{O}_{2}^{-\bullet}$ and $\mathrm{H}_{2} \mathrm{O}_{2}$, which then secondarily interacted to produce short-lived, highly pernicious radicals, such as $\mathrm{OH}^{\bullet}$, which can have a devastating effect on cell function. In this study we have used the scavenging enzymes superoxide dismutase and catalase to selectively remove $\mathrm{O}_{2}{ }^{-\bullet}$ and $\mathrm{H}_{2} \mathrm{O}_{2}$ from the reaction mixture and thereby determine their relative cytotoxicity towards human spermatozoa. Furthermore, the influence of short-lived radicals has been investigated by experiments involving the delayed addition of spermatozoa, the use of scavengers and the incorporation of transition metal chelators (DETAPAC and desferrioxamine) into the reaction medium. With the aid of these strategies we have demonstrated that reactive oxygen species can exert a direct cytotoxic effect on human spermatozoa and generated data on the nature of the oxidant involved.

The superoxide anion has been suggested as a cytotoxic species in the context of human spermatozoa and emphasis placed on the value of superoxide dismutase as a protective enzyme (Alvarez et al., 1987; Bize et al., 1991). There is certainly good evidence that in the aprotic conditions that exist in the interior of biological membranes, $\mathrm{O}_{2}{ }^{-\bullet}$ can act as a powerful nucleophile, inducing the de-esterification of membrane phospholipids (Deby et al., 1990). Furthermore, $\mathrm{O}_{2}^{-\bullet}$ has been invoked 
as a participant in the Haber-Weiss cycle, through its capacity to reduce the transition metals (iron and copper) that act as catalysts for $\mathrm{OH}^{\bullet}$ formation in this series of reactions. However, the data presented in this paper indicate that although such theoretical possibilities exist, $\mathrm{O}_{2}{ }^{-\bullet}$ does not appear to exert a cytotoxic effect on human spermatozoa. Thus, the ability of the xanthine oxidase system to disrupt both the motility of human spermatozoa and their capacity for sperm-oocyte fusion was not alleviated by the elimination of $\mathrm{O}_{2}^{-} \bullet$ from the reaction mixture with SOD. Moreover, SOD has been shown to be incapable of inhibiting ferrous ion promoted lipid peroxidation (Mennella and Jones, 1980) in mammalian spermatozoa, suggesting that this enzyme might be of limited use to these cells in their defence against oxidative stress. In view of the low concentrations of catalase associated with mammalian spermatozoa, the presence of SOD might even be considered deleterious since the product of dismutation $\left(\mathrm{H}_{2} \mathrm{O}_{2}\right)$ is more harmful than $\mathrm{O}_{2}^{-}-$itself. Much would depend on the effectiveness of glutathione peroxidase in eliminating $\mathrm{H}_{2} \mathrm{O}_{2}$ from the spermatozoa. Human spermatozoa certainly contain this enzyme system (Alvarez and Storey, 1989), although its significance in the defence of human spermatozoa against oxidative stress has not yet been fully elucidated.

In contrast to SOD, the addition of catalase to the incubation system completely reversed the cytotoxic effect of the xanthine oxidase system, whether sperm movement or sperm-oocyte fusion was considered as the biological end-point. In the presence of this enzyme $\mathrm{H}_{2} \mathrm{O}_{2}$ is eliminated and converted to oxygen and water. Moreover, catalase enhances the generation of $\mathrm{O}_{2}^{-\bullet}$, by preventing the suicidal destruction of xanthine oxidase via mechanisms that involve the direct transfer of electrons from the enzyme to $\mathrm{H}_{2} \mathrm{O}_{2}$ and consequent site-directed $\mathrm{OH}{ }^{\bullet}$ formation (Terada et al., 1991). The protective effect of catalase in these experiments therefore suggests that it is exclusively $\mathrm{H}_{2} \mathrm{O}_{2}$ that can exert a direct cytotoxic effect on human spermatozoa, rather than $\mathrm{O}_{2}^{-} \bullet$. Although $\mathrm{H}_{2} \mathrm{O}_{2}$ could theoretically participate in secondary reactions, leading to $\mathrm{OH} \bullet$ formation through the Haber-Weiss cycle or the peroxidase-mediated generation of hypochlorous acid, these possibilities were excluded as far as possible by the use of scavengers (mannitol and taurine) and transition metal chelators (DETAPAC and desferrioxamine). Furthermore, the cytotoxic species generated in the xanthine oxidase system were shown to be extremely stable, unlike the short-lived radical species such as $\mathrm{OH}^{\bullet}$, and there is good data to suggest that, in the absence of added iron, the xanthine oxidase system cannot generate $\mathrm{OH}^{\bullet}$ even in the absence of chelating and scavenging agents (Link and Riley, 1988; Britigan et al., 1990). It therefore seems unlikely that $\mathrm{OH}^{\bullet}$ or $\mathrm{O}_{2}^{-\bullet}$ arising from the xanthine oxidase system could have been directly involved in the initiation of peroxidative damage. However, this does not exclude the possibility that lipid peroxidation can be initiated within the microenvironment of the sperm plasma membrane by oxygen radicals generated from $\mathrm{H}_{2} \mathrm{O}_{2}$ in a Fenton reaction.

A direct suppressive effect of $\mathrm{H}_{2} \mathrm{O}_{2}$ on sperm movement and the capacity of these cells to acrosome react and fuse with the vitelline membrane of the oocyte confirmed the cytotoxicity of this oxidizing species towards human spermatozoa. The pathology induced by exposure to low concentrations of $\mathrm{H}_{2} \mathrm{O}_{2}$, characterized by a reduced capacity to exhibit the acrosome reaction and sperm-oocyte fusion in response to A23187, parallels the situation frequently observed in patients (Aitken et al., 1984, 1989b, 1992a; Cummings et al., 1991). In such cases, the inability of spermatozoa to exhibit biological responses following stimulation with A23187 is frequently associated with enhanced luminol-dependent chemiluminescence, reflecting the excessive generation of reactive oxygen species (Aitken and Clarkson, 1987a,b; Aitken et al., 1989b, 1992a). Moreover, studies have indicated that the major oxidant detected by luminoldependent chemiluminescence is $\mathrm{H}_{2} \mathrm{O}_{2}$ (Aitken et al., 1992b). Although spermatozoa do possess cytoplasmic enzymes for scavenging $\mathrm{H}_{2} \mathrm{O}_{2}$ (Alvarez and Storey, 1989; Jeulin et al., 1989), the cytoplasm of the human spermatozoon is extremely limited in terms of its volume and localization, with the result that the unsaturated fatty acids that abound in the sperm plasma membrane are very susceptible to peroxide attack.

The mechanism by which $\mathrm{H}_{2} \mathrm{O}_{2}$ mediates its cytotoxic effect is currently unknown. Disruption of calcium metabolism is one possibility, since the pathological effects of ROS on endothelial cells have been shown to involve an increase in the intracellular concentration of this cation (Hirosumi et al., 1988). However, because the arrest of sperm movement in the presence of the xanthine oxidase system was not curtailed by the omission of calcium from the medium, or the addition of EGTA (R. J. Aitken, unpublished), changes in intracellular calcium are probably not directly involved in the oxidant-induced loss of sperm function. There are, of course, many other ways in which the physical and chemical changes induced in the sperm plasma membrane by $\mathrm{H}_{2} \mathrm{O}_{2}$ might have impaired the functional competence of these cells. Peroxide-induced increases in membrane order and rigidity (Block, 1991) could have had a direct effect on the ability of the spermatozoa to initiate the membrane fusion events associated with the acrosome reaction and fusion with the oocyte. At higher doses, $\mathrm{H}_{2} \mathrm{O}_{2}$-induced lipid peroxidation might have disrupted the integrity of the plasma membrane, through the activation of phospholipase $A_{2}$ and the consequential loss of fatty acids from this structure (Ungemach, 1985). A variety of membrane-bound and mitochondrial enzyme systems, for example $\mathrm{Ca}^{2+}$-ATPases (Ohta et al., 1989), are also possible targets for peroxide-mediated damage to spermatozoa and could be examined in future studies of defective human spermatozoa.

Hydrogen peroxide has been shown to be a toxic species for many disparate cell types (Nathan et al., 1979; Link and Riley, 1988) and although a previous investigation suggested that human spermatozoa might be relatively resistant to this particular oxidant (Wales et al., 1959) these studies were carried out in the presence of seminal plasma, which is known to exhibit antioxidant activity (Jones et al., 1979). The identification of this particular oxidant as a toxic species in the case of human spermatozoa will have important consequences in terms of the design of diagnostic tests of human sperm function and the optimization of culture conditions for the treatment of male factor infertility using in vitro fertilization procedures. In terms of diagnostics, recent studies have demonstrated that the chemiluminescent signals generated by human spermatozoa are predictive of the fertilizing capacity of these cells in vivo and in vitro (Aitken and Clarkson, 1987a,b; Aitken et al., 1989a, 1989b, 1991, 1992a). The fact that these chemiluminescent responses have been shown to reflect the generation of $\mathrm{H}_{2} \mathrm{O}_{2}$ by human 
spermatozoa (Aitken et al., 1992b) is in keeping with the results obtained in the study described here and will serve to focus attention on the development of sensitive techniques to detect this oxidant for diagnostic purposes. This susceptibility to $\mathrm{H}_{2} \mathrm{O}_{2}$ might also be important in terms of the potential that leucocytes possess for creating oxidative stress in the washed human ejaculate (Aitken and West, 1990; Kessopoulou et al., 1992) in view of previous studies indicating that the cytotoxic effect of macrophages and granulocytes on a variety of cell types is mediated by $\mathrm{H}_{2} \mathrm{O}_{2}$ (Nathan et al., 1979). In terms of therapeutics, the finding that $\mathrm{H}_{2} \mathrm{O}_{2}$ is cytotoxic to human spermatozoa, together with recent data suggesting that this oxidant is also cytotoxic to embryos (Nasr-Esfahani et al., 1990a, b), suggests an important role for $\mathrm{H}_{2} \mathrm{O}_{2}$ scavenging systems in the design of improved in vitro fertilization media.

\section{References}

Aitken RJ and Clarkson JS (1987a) Generation of reactive oxygen species by human spermatozoa. In Free Radicals: Recent Developments in Lipid Chemistry, Experimental Pathology and Medicine Pp 333-335. Eds T Dormandy and C Rice-Evans. Richelieu Press, London

Aitken RJ and Clarkson JS (1987b) Cellular basis of defective sperm function and its association with the genesis of reactive oxygen species by human spermatozoa Journal of Reproduction and Fertility 81 459-469

Aitken RJ and Elton RA (1984) Significance of Poisson distribution theory in analysing the interaction between human spermatozoa and zona-free hamster oocytes Journal of Reproduction and Fertility 72 311-321

Aitken RJ and Elton RA (1986) Application of a Poisson-gamma model to study the influence of gamete concentration on sperm-oocyte fusion in the zonafree hamster egg penetration test Journal of Reproduction and Fertility $\mathbf{7 8}$ 733-739

Aitken RJ and West KM (1990) Analysis of the relationship between reactive oxygen species production and leucocyte infiltration in fractions of human semen separated on Percoll gradients Intemational Journal of Andrology 13 433-451

Aitken RJ, Ross A, Hargreave T, Richardson D and Best F (1984) Analysis of human sperm function following exposure to the ionophore A23187: comparison of normospermic and oligozoospermic men. Journal of Andrology 5 321-329

Aitken RJ, Clarkson JS and Fishel S (1989a) Generation of reactive oxygen species, lipid peroxidation and human sperm function Biology of Reproduction $40183-197$

Aitken RJ, Clarkson JS, Hargreave TB, Irvine DS and Wu FCW (1989b) Analysis of the relationship between defective sperm function and the generation of reactive oxygen species in cases of oligozoospermia Joumal of Andrology 10 214-220

Aitken RJ, Irvine DS and Wu FC (1991) Prospective analysis of sperm-oocyte fusion and reactive oxygen species generation as criteria for the diagnosis of infertility American Joumal of Obsterrics and Gynecology 164 542-551

Aitken RJ, Buckingham D, West K, Wu FC, Zikopoulos K and Richardson DW (1992a) Differential contribution of leucocytes and spermatozoa to the high levels of reactive oxygen species recorded in the ejaculates of oligozoospermic patients Joumal of Reproduction and Fertility 94 451-462

Aitken RJ, Buckingham DW and West KM (1992b) Reactive oxygen species and human spermatozoa; analysis of the cellular mechanisms involved in luminoland lucigenin-dependent chemiluminescence Joumal of Cellular Physiology $151466-477$

Aivarez JG and Storey BT (1989) Role of glutathione peroxidase in protecting mammalian spermatozoa from loss of motility caused by spontaneous lipid peroxidation Gamete Research 23, 77-90

Alvarez JG, Touchstone JC, Blasco L and Storey BT (1987) Spontaneous lipid peroxidation and production of hydrogen peroxide and superoxide in human spermatozoa Journal of Andrology 8 338-348

Biggers JD, Whitten WK and Whittingham DG (1971) The culture of mouse embryos in vitro. In Methods in Mammalian Embryology pp 86-94 Ed. JC Daniel. San Francisco, Freeman
Bize I, Santander G, Cabello P, Driscoll D and Sharpe C (1991) Hydrogen peroxide is involved in hamster sperm capacitation in vitro Biology of Reproduction 44 398-403

Block ER (1991) Hydrogen peroxide alters the physical state and function of the plasma membrane of pulmonary artery endothelial cells Journal of Cellular Physiology 146 362-369

Britigan BE, Pou S, Rosen GM, Lilleg DM and Buettner GR (1990) Hydroxyl radical is not a product of the reaction of xanthine oxidase and xanthine Joumal of Biological Chemistry 265 17533-17538

Cummings JM, Pember SM, Jequier AM, Yovich JL and Hartmann PE (1991) A test of the human sperm acrosome reaction following ionophore challenge (ARIC) Joumal of Andrology 12 98-103

Deby C, Boes M, Pincemail J, Bourdon-Neuray J and Deby-Dupont G (1990) Degradation of membrane phospholipids by a direct nucleophilic action of superoxide anion. In Free Radicals, Lipoproteins and Membrane Lipids pp 105-114 Eds A Crastes de Paulet, L Douste-Blazy and R Paoletti. Plenum Press, New York

Green MJ and Hill HAO (1984) Chemistry of dioxygen Methods in Enzymology $1053-22$

Gyllenhammar H (1987) Lucigenin chemiluminescence in the assessment of neutrophil superoxide production Journal of Immunological Methods 97 209-213

Hirosumi J, Ouchi Y, Watanabe M, Kusunoki J, Nakamura T and Orimo H (1988) Effect of superoxide and lipid peroxide on cytosolic free calcium concentration in cultured pig aortic endothelial cells Biochemical and Biophysical Research Communications 152 301-307

Hull MGR, Glazener CMA, Kelly NJ, Conway DI, Foster PA, Hunton RA, Coulson C, Lambert PA, Watt EM and Desai KM (1985) Population study of causes, treatment and outcome of infertility British Medical Journal 291 1693-1697

Iwasaki A and Gagnon C (1992) Formation of reactive oxygen species in spermatozoa of infertile patients Fertility and Sterility 57 409-416

Jeulin C, Soufir JC, Weber P, Laval-Martin D and Calvayrac R (1989) Catalase activity in human spermatozoa and seminal plasma Gamete Research 24 185-196

Jeyendran RS, Van der Ven HH, Perez-Palaez M, Crabo BG and Zaneveld LJD (1984) Development of an assay to assess the functional integrity of the human sperm membrane and its relationship to other semen characteristics Journal of Reproduction and Fertility 70 219-228

Jones R, Mann T and Sherins RJ (1979) Peroxidative breakdown of phospholipids in human spermatozoa: spermicidal effects of fatty acid peroxides and protective action of seminal plasma Fertility and Sterility 31 531-537

Kessopoulou E, Tomlinson MJ, Barratt CLR, Bolton AE and Cooke ID (1992) Origin of reactive oxygen species in semen; spermatozoa or leukocytes? Journal of Reproduction and Fertility 94 463-470

Lessley BA and Garner DL (1983) Isolation of motile spermatozoa by density gradient centrifugation in Percoll Gamete Research 7 49-61

Link EM and Riley PA (1988) Role of hydrogen peroxide in the cytotoxicity of the xanthine/xanthine oxidase system Biochemical Joumal 249 391-399

McCord JM and Fridovich I (1968) The reduction of cytochrome $c$ by milk xanthine oxidase Joumal of Biological Chemistry $2435733-5760$

Mennella MRF and Jones R (1980) Properties of spermatozoal superoxide dismutase and lack of involvement of superoxides in metal-ion-catalysed lipidperoxidation reactions in semen Biochemical Journal 191 289-297

Mortimer D, Curtis EF and Miller RG (1988) Specific labelling by sperm agglutinin of the outer acrosomal membrane of the human spermatozoon Journal of Reproduction and Fertility 81 127-135

Nasr-Esfahani M, Aitken RJ and Johnson MH (1990a) The measurement of $\mathrm{H}_{2} \mathrm{O}_{2}$ levels in preimplantation embryos from blocking and non-blocking strains of mice Development 109 501-507

Nasr-Esfahani M, Johnson M and Aitken RJ (1990b) The effect of iron and iron chelators on the in vitro block to development of the mouse preimplantation embryo: BTT6 a new medium for improved culture of mouse embryo in vitro Human Reproduction 5 997-1003

Nathan CF, Silverstein SC, Brukner LH and Cohn ZA (1979) Extracellular cytolysis by activated macrophages and granulocytes joumal of Experimental Medicine 149 100-113

Ohta A, Mohri T and Ohyashiki T (1989) Effect of lipid peroxidation on membrane-bound $\mathrm{Ca}^{2+}$-ATPase activity of the intestinal brush-border membranes Biochemica Biophysica Acta 984 151-157

Radi R, Rubbo H, Thomson $\mathbf{L}$ and Prodanov E (1990) Luminol chemiluminescence using xanthine and hypoxanthine as xanthine oxidase substrates Free Radicals in Biology and Medicine 8 121-126 
Slater TF (1984) Overview of methods used for detecting lipid peroxidation Methods in Enzymology 105 283-292

Terada LS, Leff JA, Guidot DM, Willingham IR and Repine JE (1991) Inactivation of xanthine oxidase by hydrogen peroxide involves site-directed hydroxyl radical formation Free Radicals in Biology and Medicine 10 61-68

Ungemach FR (1985) Plasma membrane damage to hepatocytes following lipid peroxidation: involvement of phospholipase $\mathrm{A}_{2}$. In Free Radicals in Liver Injury pp 127-134 Eds G Poli, KH Cheeseman, MU Dianzani and TF Slater. IRL Press, Washington

Vilim V and Wilhelm J (1989) What do we measure by a luminol-dependent chemiluminiscence of phagocytes? Free Radicals in Biology and Medicine 6 $623-628$
Wales RG, White IG and Lamond DR (1959) The spermicidal activity of hydrogen peroxide in vitro and in vivo Journal of Endocrinology 18 236-244 Wolff HG and Anderson DJ (1988a) Immunohistologic characterization and quantitation of leucocyte subpopulations in human semen Fertility and Sterility 49 497-504

Wolff $\mathbf{H}$ and Anderson DJ (1988b) Evaluation of granulocyte elastase as a seminal plasma marker for leukocytospermia Fertility and Sterility 50 129-132

World Health Organization (1987) WHO Laboratory Manual for the Examination of Human Semen and Semen-Cervical Mucus Interaction. Cambridge University Press, Cambridge

Wymann MP, von Tscharner V, Deranleau DA and Baggiolini M (1987) Chemiluminescence detection of $\mathrm{H}_{2} \mathrm{O}_{2}$ produced by human neutrophils during the respiratory burst Analytical Biochemistry $165371-378$ 